\title{
Alcohol Use in Patients After Liver Transplantation for Alcoholic Liver Cirrhosis Detected by the Questionnaire (Regional Experience)
}

\author{
Pavel Wohl ${ }^{*}, 1$, Petr Wohl ${ }^{2}$, Pavel Trunečka ${ }^{1}$, Jan Šperl ${ }^{1}$, Eva Honsová ${ }^{3}$ and Julius Špičák ${ }^{1}$ \\ ${ }^{I}$ Department of Hepatogastroenterology, ${ }^{2}$ Diabetes Center, ${ }^{3}$ Department of Pathological Anatomy, Institute for Clinical \\ and Experimental Medicine (IKEM), Prague, Czech Republic
}

\begin{abstract}
Background: Alcoholic liver cirrhosis is a common indication for liver transplantation. Recurrence of alcohol use was retrospectively analyzed after liver transplantation.

Methods: From X/1995 to II/2002, 42 out of 246 patients underwent liver transplantation for alcoholic liver cirrhosis. We administered a questionnaire to all patients surviving at least 1 year post liver transplantation for alcoholic liver cirrhosis. Liver biopsy was performed annually. The results of the questionnaires of both alcohol use and sobriety group were compared with laboratory parameters, graft histopathology, socioeconomic status and survival rate.

Results: Sixteen patients admitted alcohol consumption in the questionnaire. Serious graft damage was diagnosed in 4 recipients, 2 of them died due to graft failure, one for another reason, 12 returned to alcohol use. We have found more steatosis, presence of Mallory hyaline and no difference in fibrosis between alcohol use and sobriety group. The alcohol use and sobriety group differed in body mass index, triacylglycerols, medium corpuscular volume and activity of $\gamma$ glutamyltransferase. Cumulative survival rate [1,3,5 years] was significantly lower in alcohol use group than in sobriety group.

Conclusion: Patients after liver transplantation for alcoholic liver cirrhosis with alcohol use had lower survival rate as compared with sobriety group. Considering the differences in histopathology and laboratory parameters in patients with and without alcohol use after liver transplantation, administration of questionnaires seems to be a valid tool for recurrence of alcohol use detection.
\end{abstract}

Keywords: alcoholic liver disease, alcohol use, cirrhosis, liver transplantation.

\section{INTRODUCTION}

Although alcoholic liver disease (ALD) as an indication for liver transplantation (OLT) is indisputable, it deserves special attention. Patients with ALD indicated for OLT are selected using strict criteria to minimize the risk of recurrent alcohol use. The number of patients diagnosed with ALD increases and long-term total abstinence is the basic prerequisite for inclusion into the waiting list for OLT. Despite the strict and comprehensive inclusion criteria, alcohol use recurs in $10-50 \%$ of patients, resulting in severe liver graft damage in $10 \%$ of cases [1-5].

The definition of alcohol drinking after OLT is disputable. "Relapse" or "recidivism" is often published in literature [6, 7]. In studies of alcohol use after OLT, "relapse "is generally defined as any alcohol use. We have used to term "alcohol use (AU)" which is any intake of alcohol after OLT. Alcohol use and dependence are diagnosed for which are established diagnostic criteria [8]. Alcohol use can be identified using laboratory tests such as Aspartatetransaminase (AST): alanin-aminotransferase (ALT) ratio, GMT gamaglutamyl transferase (GMT), medium corpuscular volume (MCV) and triglyceride level determination.

*Address correspondence to this author at the Department of Hepatogastroenterology, Institute for Clinical and Experimental Medicine, Vídeňská 1958, Prague 4, Czech Republic; Tel: + 42026136 2139;

Fax: + 42026136 2697; E-mail: pawo@ikem.cz
However, these tests are commonly biased by other posttransplantation factors. Thus, it is either the patient's acknowledgement or the finding of alcohol or its metabolites in his/her blood that provide unequivocal evidence of alcohol use. In our study, a structured-questionnaire method was used to detect alcohol use recurrence in patients after OLT.

The goal of this retrospective study was to evaluate the course of post-transplant treatment of liver transplant recipients in terms of alcohol use recurrence.

\section{SUBJECTS}

From October 1995 through February 2002, 246 patients with ALD were indicated for OLT in the transplant center at the Institute for Clinical and Experimental Medicine (IKEM) in Prague, Czech Republic. Of this number, 42 (17.1\%) were diagnosed with ALD and $30(6.4 \%)$ with liver cirrhosis of mixed etiology (for example hepatitis and use alcohol) and others), which were excluded from study. Only patients with at least one liver graft biopsy sample obtained at least one year after OLT were eligible for inclusion into the study. All patients gave their informed consent prior to their inclusion in the study. P.S.: This study was performed in accordance with the ethical standards laid down in the 1964 declaration of Helsinki.

\section{METHODS}

Any acknowledged alcohol consumption in the questionnaire alcohol use recurrence was regarded as alcohol 
Table 1. Post-Transplant Alcohol Use Questionnaire at Out Hepatogastroenterology Department (IKEM)

1. Do you often have appetite for an alcoholic beverage?

[1] Yes [0] No

2. Have you visited a psychologist, a psychiatrist, or an anti-toxic [AT] counselor after the transplantation?

[1] Yes [0] No

3. Have you ever drunk an alcoholic drink after the liver transplantation?

[1] Yes [0] No

4. How often do you drink alcoholic beverages?
[1] Monthly
[2] 2-4 times a month [3] 2-3 times a month [4] 4 + more times a month

5. Do you drink daily or did you have a period of daily drinking after the transplant?

[1] Yes [0] No

6. What alcoholic beverages do you drink and how many glasses do you drink?
[1] beer $[0.51$ glass $]$.......
[2] wine [0.21 glass] .......
[3] spirit [0.051 glass] .......
[4] combination

use (Table 1). The main and only criterion for alcohol use was characterized as any alcohol intake indicating for alcohol use as well as this was also used as our definition. We prefer any intake as alcohol use because our country is leading in drinking habits as compared to other countries. This questionnaire was prepared by psychiatric with view to alcohol addiction and our hepatology teams.

All patients underwent psychological a psychiatric evaluation before OLT. Six month abstinence period is strictly keeping before OLT in majority of transplant centers. One justification of the period of abstinence before OLT has been to allow patients with acute alcoholic hepatitis the opportunity to recover with medical management. Up to now, patients with recent alcohol use have been precluded from OLT. But, we lack good data to determine whether some of these patients would benefit from OLT [9]. In our centre we have significantly required 6 months abstinence before OLT.

The following measures were evaluated as parameters: gammaglutamyl-tranferase (GMT), mean erythrocyte volume (MCV), triglycerides (TAG), and body mass index (BMI). Liver graft function was assessed using serum albumin level and prothrombin time. Liver graft biopsies were reevaluated semi quantitatively from the point of view of occurrence of liver steatosis, Mallory hyaline and fibrosis. The survival rates of patients and liver grafts in the above groups at 1,3 , and 5 years after the transplantation were compared using the Kaplan-Meier curve. Student's t-test, the $\chi^{2}$-test (Pearson) and Fischer's exact test were used for statistical analysis. Only first transplant procedures were included in the study.

\section{RESULTS}

Thirty-eight out of 42 patients (90\%) completed the questionnaire (Table 2), four patients did not respond, and three patients died with unequivocally established alcohol use (acknowledgement, alcohol found in blood). Thirteen patients acknowledged alcohol use recurrence in the questionnaire.

No patient acknowledged daily alcohol consumption. Nine patients with acknowledged alcohol use and one patient declaring abstinence underwent psychological/psychiatric investigation. Eight patients acknowledged appetite for an alcoholic drink, all from the group with confirmed alcohol use. Patients who acknowledged alcohol use usually declared alcohol intake 4 and more times a month. The most common alcoholic beverage was beer. Three patients who died with unequivocally established alcohol use were included into the group which acknowledged alcohol consumption in the questionnaire.

Table 2. Questionnaire Results

\begin{tabular}{|l|c|c|c|c|}
\hline \multirow{2}{*}{ Patients Questions } & \multicolumn{2}{|c|}{ Alcohol Use } & \multicolumn{2}{c|}{ Abstinence } \\
\cline { 2 - 5 } & Yes & No & Yes & No \\
\hline \hline 1. Appetite for an alcoholic drink? & 8 & 5 & 0 & 22 \\
\hline 2. AT counselor visit after OLT? & 9 & 4 & 2 & 20 \\
\hline 3. Alcohol use after OLT? & 13 & 0 & 0 & 22 \\
\hline
\end{tabular}

Among the 42 patients who underwent OLT for ALD, alcohol use was found in 16 (38\%) patients (Table 3) In 4, alcohol use was accompanied by the development of liver graft dysfunction; of this number, 2 patients died of liver graft failure due to alcohol use recurrence. Another patient with substantial alcohol use refused haemodialysis therapy and died of renal failure. Twelve patients resumed alcohol use without detectable liver graft dysfunction.

Table 3. Comparison of Patients with Alcohol Use Group and Patients Declaring Abstinence $\left(\chi^{2}\right.$-Test)

\begin{tabular}{|llll|}
\hline & Alcohol Use & Declared Abstinence & p \\
\hline \hline Number & 16 & 22 & \\
\hline Age [yrs] & $47.75 \pm 7.14$ & $49.45 \pm 8.02$ & NS \\
\hline Sex [Male/Female] & $10 / 6$ & $20 / 2$ & $\mathrm{p}<0.05$ \\
\hline Education & & & \\
\hline *Elementary & 1 & 1 & \\
\hline *Secondary & 13 & 15 & $\mathrm{p}<0.05$ \\
\hline *University & 2 & 6 & $\mathrm{NS}$ \\
\hline Smoking status & 11 & 8 & $\mathrm{p}<0.07, \mathrm{NS}$ \\
\hline Divorce rate & 5 & 4 & \\
\hline Death [alcoholic] & 3 & 0 & \\
\hline *Sex smoking status a death & &
\end{tabular}


Substantial liver graft steatosis occurred in the acknowledged alcohol use group; moreover, it was only this group which showed occurrence of Mallory hyaline. The alcoholic origin of the liver graft steatosis in this group is supported by the lower incidence of risk factors such as obesity, diabetes, and $\mathrm{HCV}$, by lower BMI and higher activity of GMT, MCV, and TAG. No difference was found in the incidence of liver fibrosis (Table 4). Three patients of the acknowledged alcohol use group were diagnosed to have acute alcoholic hepatitis ( $<<0.07 ; \mathrm{NS}, \chi^{2}$-test).

Table 4. Post-Transplant Histopathologic Findings ( $\chi^{2}$-Test)

\begin{tabular}{|lllr|}
\hline & $\begin{array}{c}\text { Alcohol Use } \\
\text { [n=16] }\end{array}$ & $\begin{array}{c}\text { Declared } \\
\text { Abstinence } \\
\text { [n=22] }\end{array}$ & p \\
\hline \hline Liver graft steatosis & & & \\
\hline * Up to $10 \%$ & 4 & 14 & \\
\hline * Macroglobular up to 50\% & 3 & 1 & $* \mathrm{p}<0.05$ \\
\hline * Mixed up to 50\% & $3 *$ & 6 & \\
\hline * More than 50\% & $6 *$ & 1 & $\mathrm{p}<0.07 ; \mathrm{NS}$ \\
\hline Mallory hyaline & $3 *$ & 0 & \\
\hline Liver graft fibrosis & & & $\mathrm{NS}$ \\
\hline Perivenular + periportal fibrosis & 8 & 10 & \\
\hline Acute alcoholic hepatitis & 3 & 0 & \\
*More than 50 \%, Mallory hyaline, acute alcoholic hepatitis. & \\
\hline
\end{tabular}

Differences were noted between patients with alcohol use and patients who declared abstinence in BMI $(25.40 \pm 3.33 v s$ $27.83 \pm 3.85 ; \mathrm{p}<0.04)$, TAG $(3.73 \pm 2.07$ vs $2.36 \pm 1.76$; $\mathrm{p}<0.04)$, MCV $(97.49 \pm 7.02$ vs $87.69 \pm 3.14 ; \mathrm{p}<0.0004)$, and in GMT activity $(4.48 \pm 6.96$ vs $0.79 \pm 1.17, \mathrm{p}<0.05$; all Student's t-test). The groups also differed significantly in incidence of smoking $(p<0.05)$ and $\operatorname{sex}(p<0.05)$. No difference was found in education level, divorce rate, incidence of chronic hepatitis $\mathrm{C}$ or diabetes mellitus $\left(\chi^{2}\right.$-test, Pearson).

Survival rates of patients and liver grafts are identical in our study as no patient transplanted for alcoholic liver cirrhosis was re-transplanted. The cumulative one-, three-, and five-year survival rates of patients with alcohol use after OLT were lower relative to abstaining patients $(100 \%, 83 \%$, and $74 \%$ vs $100 \%$, respectively; $\mathrm{p}<0.05$, Kaplan-Meier curve). Total survival rate of patients with ALD after OLT was higher compared to other etiologies, but the difference was not significant ( $93 \%$ vs $85 \%$, NS, Table 5).

Treatment of recurrence of alcohol use after OLT was performed by standard psychiatric and psychological procedures. Four patients who were died to alcohol use after OLT denied psychiatric treatment.

\section{DISCUSSION}

Liver transplantation for ALD is a standard therapy for advanced liver cirrhosis. Alcohol use is a specific risk with all consequences including liver graft failure and death of the patient. Considering the potential consequences, any alcohol
Table 5. Survival Rate After Liver Transplantation (100\%, $83 \%$, and $74 \%$ vs $100 \%$, Respectively; $p<0.05$, Kaplan-Meier Curve)

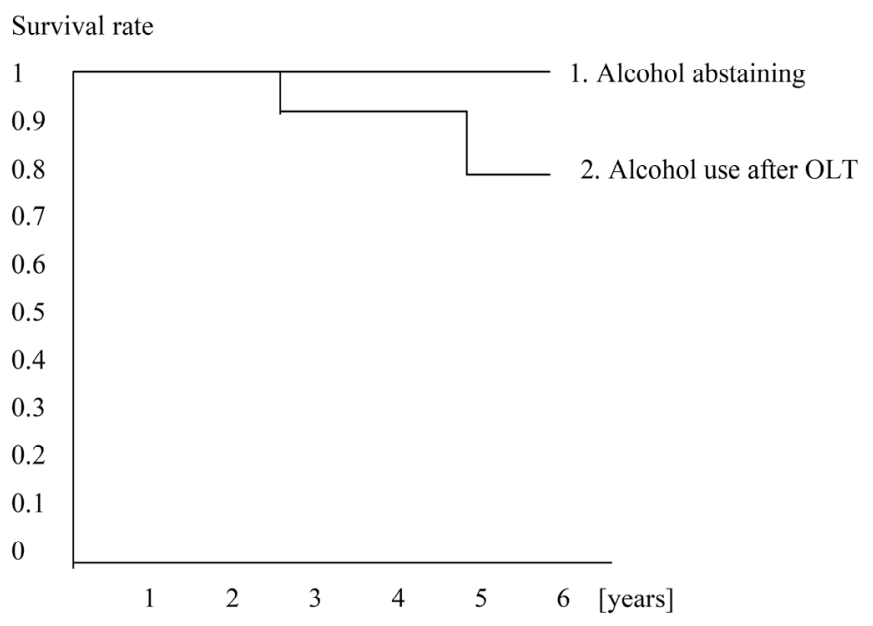

consumption after OLT is recognized as alcohol use (harmful drinking) and thus as a manifestation of alcohol addiction. The unequivocal diagnosis is established by a detailed psychiatric-psychological examination. Berlachovich et al. $[2,9,10]$ regard any alcohol consumption after OLT as alcohol use recurrence. Neuberger [1] distinguishes notions of social drinking and heavy drinkers. The importance of alcohol use detection consist lays first in timely recognition and treatment of patients with potential liver graft damage and alcohol addiction. Moderate alcohol consumption does not lead to liver graft dysfunction in most cases. Despite caveats, it makes sense to combine results from many studies and postulate that a third to half the recipient OLT for ALD start drinking again after OLT, that about $10 \%$ resume drinking in abusive or dependent fashion and that resumption of alcohol often begins within the first year after surgery [11]. Due to the risk of alcohol use recurrence, the indication of ALD for OLT was to some extent controversial in the past. At the beginning of the transplantation program, this risk was connected only with reneged 6-months abstinence period [3, 12]. Gish et al. [13] have identified risk factors of alcohol use recurrence after OLT. They assume that nondocumented abstinence, repeated failure of abstinence and anti-alcoholic therapy, low social background and psychiatric disease are the most critical risk factors. In their data of 46 patients, the most serious risk factors for alcohol use appeared to be insufficient compliance with antialcoholic therapy and psychiatric disease preceding OLT. In such a case, the median time of recurrence was 2 to 3 years.

Selection of patients with this diagnosis for inclusion into the waiting list for OLT is subject to strict criteria in order to increase probability of total abstinence. The main criteria applied at IKEM are a detailed psychiatric-psychological examination, personal contacts with physicians and a documented 6-month abstinence period. In our department, patients with ALD stand an approximately $50 \%$ chance of being included into the waiting list for OLT compared to other etiologies [14].

Despite the risk of alcohol use after OLT, the number of patients with this diagnosis indicated for OLT has been on 
the rise. In 1990, 25\% of patients included in the European Register of Liver Transplantation had the above diagnosis. In 1996, the figure was as high as 40\%. Between 1968 and 1995, 2480 liver transplant procedures for ALD were carried out [i.e., $12 \%$ of all liver transplant procedures]. In this study, alcohol use was detected in $28 \%$ of patients [5-60\%] [1]. Other authors have reported alcohol use rates of $10-49 \%$ $[1-3,12]$. Using a questionnaire before and after OLT, Osorio et al. [12] examined 43 patients with ALD. In their study, the despondence rate was $86 \%$; of this number, $19 \%$ acknowledged alcohol use after OLT.

In earlier studies [15], the survival rates of patients with ALD after OLT were lower than that of patients with other etiologies. Currently, a number of studies have shown that the survival rate of patients with this etiology is higher or equivalent to other etiologies [1]. In the study of Pageaux et al. [16], the survival rate of patients with ALD did not differ from the rate with other etiologies. Bellamy et al. [9] showed no influence of alcohol use recurrence on liver graft damage or failure. On the other hand, they report a higher incidence of malignancies, especially of otolaryngological and bronchial/pulmonary origin, which correlates with the recipients' higher morbidity and mortality rates. These findings are related to the frequent incidence of chronic nicotinism in alcoholics. We have no neoplasm "de novo" in our population after OLT for ALD during 7 years. Our study has some limitations. The main limitation is a small group of tested subjects. The questionare that we have used has not been validated for the large population, thus our results could not be considered as generalize conclusion. However our method might be a suitable tool for alcohol use reccurence diagnosis in specific patients after liver transplantation. In addition, precize evaluation is mandatory for OLT patients, therefore our main criterium have been chosen as positive ," any alcohol intake" to detected the alcohol use highest number of patients. General psychiatric diagnostic methods are often inconclusive in alcohol use transplant patients, because OLT pateints are the specific group. Because the liver candidate selection procedure as well as costeffectiveness evaluation of OLT treatment might be responsible for this specificity. Unfortunately, the analysis dealing with the comparison of selected diagnostic alcohol use methods has not been our aim.

Clinical analysis and repeated outpatient examinations may lead to a suspicion for alcohol use in patients after OLT. However, unequivocal evidence is provided by the patients' acknowledgement or by direct detection of alcohol in the patient's blood or body fluids.

Detection of alcohol use recurrence after OLT is difficult. Generally, $50 \%$ of patients with alcohol use recurrence are identified on their first visit, but moderate forms may remain concealed for long [17]. Apart from biochemical laboratory tests, which can yield false positive as well as false negative results, our study used the questionnaire [12]. Standard alcohol use markers such as GMT, MCV, or AST/ALT ratio may serve as inaccurate measures of alcohol use. They can be influenced by other factors such as obesity, immunosuppressive therapy, or hyperlipoproteinemia.
A Viennese group [18] considers carbohydrate-deficient transferrin [CDT] a reliable method for detecting posttransplant alcohol use with $92 \%$ sensitivity and $98 \%$ specificity. By contrast, Sorvajarvi et al. [19] did not confirm the reliability of CDT. Berlakovich et al. [10] used immunosuppressive [cyclosporin] levels as a measure of compliance with therapy.

A histopathological examination of the liver graft is another possibility of detecting alcohol-induced changes. Birds et al. [20] found changes [Mallory hyaline, steatosis, fibrosis] corresponding to toxic alcoholic damage in patients with acknowledged alcohol use group. Our study also revealed a significant difference in the incidence of Mallory hyaline and liver graft steatosis between the groups of patients with acknowledged alcohol use group and declared abstinence. The incidence of liver steatosis may indicate recent alcohol use as it is reported to subside after approximately two months of abstinence.

Sixteen patients of our group acknowledged alcohol use. The clinical suspicion of alcohol use correlated with the questionnaire-based findings in 9 cases. The questionnaire revealed 7 new patients with alcohol use. The severity of liver graft steatosis, Mallory hyaline and laboratory and additional examinations correlated in patients with acknowledged alcohol use. A suspicion for alcohol use was also raised in 4 patients with a higher incidence of liver steatosis who did not respond to the questionnaire. In our study we had used a questionnaire with respect to our national population and Czech habits of alcohol drinking can be probably different in contrast to western world. We suppose that it may have great influence to results of our study.

\section{CONCLUSION}

Patients after liver transplantation for alcoholic liver cirrhosis with alcohol use show lower survival rates compared to those of patients with declared abstinence. Alcohol use after OLT may be fatal. Its incidence in our series corresponds to findings in the relevant literature.

\section{ACKNOWLEDGEMENT}

Declared none.

\section{CONFLICT OF INTEREST}

Declared none.

\section{ABBREVIATIONS}

$\mathrm{AU}=$ Alcohol use
$\mathrm{SG}=$ Sobriety group
$\mathrm{OLT}=$ Orthotopic liver transplantation
$\mathrm{ALD}=$ Alcohol liver disease
REFERENCES

[1] Neuberger J. Transplantation for alcoholic liver disease. A perspective from Europe. Liver Transplant Surg 1998; 4: 51-7.

[2] Berlakovich GA, Steininger R, Hebst F, Barlan M, Mittlbock M, Muhlbacher F. Efficacy of liver transplantation for alcoholic liver cirrhosis with respect to recidivism and compliance. Transplantation 1994; 58: 560-7. 
[3] Kumar S, Stauber RE, Gevaler JS. Orthotopic liver transplantation for alcoholic liver disease. Hepatology 1990; 11(1): 159-64.

[4] Tome S, Lucey MR. Timing of liver transplantation in alcoholic cirrhosis. J Hepatol 2003; 39: 302-7.

[5] Mackie J, Groves K, Hoyle A, et al. Orthotopic liver transplantation for alcoholic liver disease: a retrospective analysis of survival, recidivism, and risk factors predisposing to recidivism. Liver Transplant 2001; 7: 418-27.

[6] Pereira SP, Williams R. Alcohol relapse and functional outcome after liver transplantation for alcoholic liver disease. Liver Transplant 2001; 7:204-5.

[7] Pageaux GP, Bismuth M, Perney P, et al. Alcohol relapse after liver transplantation for alcoholic liver disease: does it matter? $\mathrm{J}$ Hepatol 2003; 38: 629-34.

[8] Neuberger J, Schulz KH, Day C et al. Transplantation for alcoholic liver disease. J Hepatol 2002; 36: 130-7.

[9] Bellamy CO, DiMartini AM, Ruppert K, Jain A, Dodson F, Torbeson M. Liver transplantation for alcoholic cirrhosis: long term follow-up and impact of disease recurrence. Transplantation 2001; 72: 619-26.

[10] Berlakovich GA, Langer F, Freunddorfer E, et al. General compliance after liver transplantation for alcoholic cirrhosis. Transpl Int 2000; 13: 129-35.

[11] Tang H, Boulton R, Gunson B, Hubscher S, Neuberger J. Pattern of alcohol consumption after liver transplantation. Gut 1998; 43: 1405 .

[12] Osorio RW, Ascher NL, Avery M. Predicting recidivism after orthotopic liver transplantation for alcoholic liver disease. Hepatology 1994; 20: 105-10.
[13] Gish RG, Lee A, Brooks L, Leung J, Lau JYN, Moore DH. Longterm follow-up of patients diagnosed with alcohol dependence or alcohol abuse who were evaluated for liver transplantation. Liver Tranplant 2001; 7: 581-7.

[14] Wohl P, Kostolná E, Špičák J, et al. Evaluation of the selection process of liver transplant candidates with alcoholic liver cirrhosis in IKEM, Prague. J Hepatol 2001; 34(Suppl.1): 46.

[15] Scharschmidt BF. Human liver transplantation: analysis of data on 540 patients from four centres. Hepatology 1984; 4: 95-101.

[16] Pageaux GP, Michel J, Coste P, Perney P, Possoz P, Perrigault PF. Alcoholic cirrhosis is good indication for liver transplantation, even for cases of recidivism. Gut 1999; 45(3): 421-6.

[17] McCullough AJ. Schiff's diseases of the liver. $8^{\text {th }}$ ed. Philadelphia: Lippincott Raven Press 1999; pp. 941-71.

[18] Berlakovich GA, Windhager T, Freundodorfer E, Lesch O, Steininnger R, Muhlbacher F. Carbohydrate deficient transferrin for detection of alcohol relapse after orthotopic liver transplantation for alcoholic cirrhosis. Transplantation 1999; 67: 1231-5.

[19] Sorvajarvi K, Blake JE, Israel Y, Niemela O. Sensitivity and specificity of carbohydrate-deficient transferrin as a marker of alcohol abuse are significantly influenced by alterations in serum transferrin: comparison of two methods. Alcohol Clin Exp Res 1996; 20: 449-54.

[20] Bird GL, O'Grady JG, Harvey FA, Calne RY, Williams R. Liver transplantation in patients with alcoholic liver cirrhosis: selection criteria and rates of survival and relapse. BMJ 1990; 301: 15-7.

Received: February 15, 2012

Revised: March 12, 2012

Accepted: March 20, 2012

(C) Wohl et al.; Licensee Bentham Open.

This is an open access article licensed under the terms of the Creative Commons Attribution Non-Commercial License (http://creativecommons.org/licenses/ by-nc/3.0/) which permits unrestricted, non-commercial use, distribution and reproduction in any medium, provided the work is properly cited. 